\section{ECJ: a new journal for new challenges in emergency medicine}

\section{Mario Cavazza}

Emergency Department,

S. Orsola-Malpighi University Hospital, Bologna, Italy

It is well known how the scientific process is intertwined with the increasing complexity of different knowledge fields and the different instruments needed. Needless to say, this occurs in a time when available resources are much more defined and somehow limited in comparison with the past.

The medical field is one of the most complicated in the scientific world, as it involves some basic sciences: from chemistry through physics, anatomy, physiology, to all clinical and pharmacological disciplines, just to name a few.

Among all medical sciences, emergency medicine (EM) comprises several domains. Thus, in order to be effective, EM has to provide physicians with some educational principles belonging to all known medical areas and, maybe more than other specialties, it has to enhance every aspect of the knowledge process.

Emergency nurses and physicians are aware that results can be obtained only if all nontechnical aspects - i.e. how and when decisions are made - are taken into consideration, besides mere technical knowledge of the clinical process.
The information exchange between those working in the field has to be non-stop, completely open, and focused on the actual application of all the improvements made in all medical fields. Emergency doctors are the final appliers of this knowledge.

This is the mission that brought to life the Academy of Emergency Medicine and Care (AcEMC) - an open group of experts including emergency physicians, specialists from all medical fields, and some professionals from non-medical but fundamental areas contributing to emergency culture. In this perspective, communication and education experts, sociologists, psychologists, healthcare building engineers, and technology, computer and safety experts are part of AcEMC.

AcEMC is an open space based on multidisciplinary thinking - an academy, as ancient greek would name it. Multidisciplinarity is the Academy hallmark and a feature which makes it quite different from individual scientific societies.

AcEMC needed a way to confront and express itself publicly. This has been done by means of a magazine - the Emergency Care Journal (ECJ) - which can be read by all the people involved in emergency care.

Emergency Care Journal was founded seven years ago by Ivo Casagranda who, together with some other professionals, has successfully run this project so far. With PAGEPress as the new Publisher, ECJ has taken a further step in the direction of widening its readership. This is accomplished through a specific program.

First, the paper version of the magazine has been dropped. This kind of publishing is noto-
Correspondence: Mario Cavazza, Emergency Department, S.Orsola-Malpighi University Hospital, via Pietro Albertoni 15, 40138 Bologna, Italy.

Tel. +39.051.636.2111 - Fax: +39.051.636.1202

E-mail:mario.cavazza@aosp.bo.it

Key words: emergency medicine, AcEMC, Emergency Care Journal, multidisciplinarity.

Received for publication: 7 May 2013.

Accepted for publication: 7 May 2013.

This work is licensed under a Creative Commons Attribution 3.0 License (by-nc 3.0).

(C) Copyright M. Cavazza, 2013

Licensee PAGEPress, Italy

Emergency Care Journal 2013; 9:e1

doi:10.4081/ecj.2013.e1

riously expensive and can limit access to the contents, which, instead, must be granted to the largest number of emergency professionals possible.

Second, the journal's contents are now available for free, thus providing unrestricted access through the Open Access model. Indeed, only a wider and larger circulation of ideas can effectively improve the emergency care system.

Third, English language has been chosen as the only language used in contributions as it is acknowledged as the global language.

Finally, the peer-review system adopted guarantees a high-quality scientific level, as is convenient to ECJ. 